\title{
Intravenous Lipid Emulsion Does Not Augment Blood Pressure Recovery in A Rabbit Model of Metoprolol Toxicity
}

\author{
Alexander Browne • Martyn Harvey • Grant Cave
}

Published online: 31 March 2010

(C) American College of Medical Toxicology 2010

\begin{abstract}
Intravenous lipid emulsion (ILE) has been demonstrated to be an effective treatment for systemic toxicity associated with local anaesthetics and increasingly non-local anaesthetic agents of high lipophilicity. Effect for ILE has been demonstrated in animal models of propranolol poisoning; however, any benefit for ILE in more hydrophilic $\beta$-blockers remains uncertain. We determined to examine the effect of ILE on haemodynamic recovery following induction of hypotension with the relatively hydrophilic $\beta$-blocker, metoprolol. Twenty sedated, invasively monitored and mechanically ventilated adult New Zealand white rabbits underwent metoprolol infusion to mean arterial pressure (MAP) 60\% baseline. Intravenous rescue was given according to the following groups: $6 \mathrm{~mL} / \mathrm{kg}$ $20 \%$ lipid emulsion or $6 \mathrm{~mL} / \mathrm{kg} 0.9 \%$ saline solution over $4 \mathrm{~min}$. Haemodynamic parameters were obtained in $15 \mathrm{~min}$. MAP was 70 (interquartile range (IQR), 58-82) $\mathrm{mmHg}$ saline group and 79 (IQR, 72-89) $\mathrm{mmHg}$ ILE group at baseline, and 38 (IQR, 33-40) $\mathrm{mmHg}$ saline group and 41 (IQR, 40-43) mmHg ILE group, respec-
\end{abstract}

Location: Ruakura Animal Research Centre, Hamilton, New Zealand.

A. Browne

Emergency Medicine Registrar, Christchurch Hospital,

Riccarton Road,

Christchurch, New Zealand

M. Harvey

Waikato Hospital,

Pembroke Street,

Hamilton, New Zealand

G. Cave $(\bowtie)$

Hutt Hospital,

High Street,

Lower Hutt, New Zealand

e-mail: grantcave@gmail.com tively, following metoprolol infusion. No statistically significant difference between ILE and saline-treated groups was observed in MAP, or pulse rate, at any time point following rescue therapy. ILE was not effective in reversal of metoprolol-induced hypotension in this rabbit model. These findings lend inferential support for the 'lipid sink' as principal mechanism for the beneficial effect observed with ILE administration in other models of lipophilic $\beta$-blocker-induced toxicity.

Keywords Lipid emulsion · Metoprolol · Toxicity $\cdot$ Rabbit

\section{Introduction}

Intravenous lipid emulsion (ILE) therapy is now an accepted treatment for reversal of life-threatening local anaesthetic systemic toxicity $[1,2]$. There has furthermore been considerable laboratory and some clinical reporting of beneficial effect for ILE in toxicity from other lipophilic agents including calcium channel blockers $[3,4]$, tricyclic antidepressants [5-7] and $\beta$-blockers [4, 8-10]. While the exact mechanism of action for ILE is at present uncertain, three main hypotheses have been forwarded to explain the effects of ILE as antidote. First, ILE may serve to sequester lipophilic toxins into an expanded plasma lipid phase (the so called 'lipid sink'), thereby reducing free drug levels and/or enhancing toxin redistribution to less vital lipid-rich tissues. Second, bolus administration of free fatty acids and phospholipids may serve to overcome pharmacologic impediments to mitochondrial free fatty acid oxidation. Finally, ILE may effect to increased myocyte calcium concentration and thereby myocardial contractility. It is conceivable that a combination of mechanisms may contribute to final effect $[1,2]$. 
$\beta$-adrenergic blocking agents are among the most commonly prescribed medications in the USA, making up $5.9 \%$ of all medicine prescriptions [11]. When taken in overdose, $\beta$-blocker intoxication presents a formidable therapeutic challenge. Current recommendations for the treatment of $\beta$-blocker poisoning advocate infusion of catecholamines [12], calcium chloride [13], glucagon [14, 15] and extracorporeal life support [16] in instances of refractory cardiovascular collapse. More recently, high-dose insulin-glucose euglycaemia $[17,18]$ and Levosimendan [19] have been demonstrated effective in animal models and clinical cases of $\beta$-blocker toxicity.

In previous work, our group has demonstrated efficacy for ILE in reversing hypotension associated with infusion of the highly lipophilic $\beta$-blocker propranolol [10], but relative absence of effect in atenolol-induced hypotension in an inherently unstable rabbit model [20]. Given the methodologic limitations experienced with the later experiment, we determined to further evaluate ILE utilising another relatively hydrophilic $\beta$-blocker as toxin. Specifically, we sought to examine the effect of ILE in metoprololinduced shock in rabbits. We hypothesised demonstration of efficacy would infer a mechanism of action mediated by some metabolic effect for lipid. Conversely, absence of effect for ILE in metoprolol toxicity would lend inferential support to the 'lipid sink' as principal explanation for the effects of ILE in lipophilic $\beta$-blocker toxicity.

\section{Methods}

\section{Study Design}

This study was undertaken as part of a programme of work investigating the effects of ILE in hypotension induced by $\beta$-blocking agents of varying lipophilicity. The design was a prospective randomised, controlled, animal experiment evaluating ILE in a rabbit model of metoprolol-induced hypotension. Mean arterial pressure (MAP) served as the primary outcome variable. The experiment was performed at the Small Animals Laboratory Facility of the Ruakura Animal Research Centre, Hamilton, New Zealand. All study protocols were evaluated and approved by the Ruakura Animal Ethics Committee. Animal husbandry was in accordance with institutional guidelines for ethical animal research.

Sample size estimation was based on data from previous experiments in the programme $[8,10]$ and sought a post-treatment effect of 1.6 standard deviation difference in MAP (corresponding to $25 \mathrm{mmHg}$ in humans and deemed to be clinically significant). With power set at $80 \%$ and alpha level at 0.05 , this equated ten animals in each group.

\section{Animal Model}

Rabbits were raised in single sex conditions, with no chance of pregnancy. All animals had free access to food and water, in a temperature/humidity-controlled environment, with a 12-h day-night cycle. All animals were weighed to the nearest gramme on the day of the experiment, then cluster randomised according to age and sex to receive either $0.9 \%$ saline or $20 \%$ Intralipid (isotonic emulsion of $20 \%$ soybean oil in water) treatment groups. Rabbits were sedated with ketamine $(50 \mathrm{mg} / \mathrm{kg})$ and xylazine $(4 \mathrm{mg} / \mathrm{kg})$ by intramuscular injection. The marginal ear vein was cannulated and, thereafter, continuous intravenous anaesthesia instituted (ketamine $10 \mathrm{mg} / \mathrm{mL}$ and xylazine $1.5 \mathrm{mg} / \mathrm{mL}$ at $1.5 \mathrm{~mL} / \mathrm{kg} / \mathrm{h}$ infusion) and maintained for the duration of the experiment. If there was any evidence of animal distress during invasive procedures, additional $1-\mathrm{mL}$ boluses of anaesthetic solution were administered manually. Monitoring of heart rate and rhythm was carried out using a three lead electrocardiogram with continuous monitoring of standard limb lead II.

Arterial catheterisation of the central artery of the contralateral ear was performed, and invasive blood pressure was monitored using an Edwards Lifesciences pressure transducer connected in standard fashion to Hewlett Packard 78834A neonatal monitor. Animals then underwent tracheostomy formation using a $3.5-\mathrm{mm}$ internal diameter endotracheal tube advanced under direct vision and secured via taping. Ventilation was achieved using a Nuffield series 200 paediatric ventilator, which provided an inspiratory flow rate $0.25 \mathrm{~L} / \mathrm{min}$ of $100 \%$ oxygen, at 12 breaths per minute, with $5 \mathrm{~cm}$ of positive end expiratory pressure. Continuous colour change capnography was used to assess ventilatory adequacy (Pedicap end tidal $\mathrm{CO}_{2}$ detector, Nellcor Puritan Bennett Inc, Pleasanton, CA, USA), and repeated doses of vecuronium $0.1 \mathrm{mg} / \mathrm{kg}$ were administered as necessary to maintain paralysis following establishment of mechanical ventilation.

\section{Induction Toxicity}

Following a period of stabilisation (3-5 $\mathrm{min}$ ), animals were infused with $20 \mathrm{mg} / \mathrm{ml}$ metoprolol solution at $20 \mathrm{ml} / \mathrm{h}$ until a target MAP of $60 \%$ of baseline was reached. The infusion was made from crushed 50-mg metoprolol tartrate tablets dissolved in $0.9 \%$ saline solution, which was then passed through a Braun epidural filter (pore size $0.2 \mu \mathrm{m}$ (B. Braun Medical Inc, 824 Twelfth Avenue, Bethlehem, PA 18018, USA)) before being infused into the animal. The choice to extract metoprolol in this way was made on the basis of availability. Research into the haemodynamic effects of excipients on the model of toxicity suggests these would 
Table 1 Animal characteristics

\begin{tabular}{llll}
\hline & Lipid $(n=10)$ & Control $(n=10)$ & $P$ value \\
\hline Age (days) & $112(107-126)$ & $119(112-125)$ & 0.60 \\
Weight $(\mathrm{g})$ & $3,400(3,550-3,780)$ & $3,300(3,400-3,620)$ & 0.52 \\
Sex & 2 males, 8 females & 4 males, 6 females & 0.63 \\
\hline
\end{tabular}

Continuous data presented as mean (interquartile range)

either be removed by the filter or have no significant haemodynamic effect.

\section{Resuscitation Protocol}

On attaining target MAP, metoprolol infusion was discontinued. Animals in the control group subsequently received $6 \mathrm{~mL} / \mathrm{kg} \mathrm{0.9 \%}$ saline solution infused over $4 \mathrm{~min}$. Animals in the test group received $6 \mathrm{~mL} / \mathrm{kg} \mathrm{20 \%}$ Intralipid over a similar period. Haemodynamic metrics (pulse rate and MAP) were collected at baseline (nominally time zero), target MAP, immediately following completion of rescue treatment and then subsequently at 2.5 -min intervals to $15 \mathrm{~min}$. Pulse rate was recorded to the nearest beat per minute, and MAP to the nearest millimetres of mercury. At completion of the experiment, all animals were killed using pentobarbital overdose $(900 \mathrm{mg})$ as per institutional guidelines.

\section{Outcome Measures and Statistical Analysis}

The primary outcome measure was a comparison of MAP at nominated time-points following rescue treatment, with pulse rate and MAP increment additionally analysed as secondary variables of interest. Statistical analysis was performed using Graphpad Instat for Windows (version 3b, www.graphpad.com). As our previous studies demonstrated non-Gaussian distribution of MAP in toxicity, the MannWhitney test was deemed most appropriate for comparison of continuous variables. Fishers' exact and Chi-squared tests were used as appropriate for in comparison of dichotomous variables. A $p$ value of less than 0.05 was retained as statistically significant.

\section{Results}

Animal characteristics and baseline haemodynamic metrics are presented in Tables 1 and 2, respectively. No statistically significant differences were observed in any baseline parameter between groupings. Metoprolol dosing to toxicity was $87 \mathrm{mg}$ (interquartile range (IQR), 46-110) in the Intralipid group and $104 \mathrm{mg}$ (IQR, 56-145) in the control group; $p=0.73$.

All data were presented as median (interquartile range).

MAP and heart rate are presented graphically in Figs. 1 and 2, respectively. No significant difference in MAP or pulse rate was observed at any measured time-point during resuscitation or monitoring thereafter (Table 2). Similarly, no difference in MAP increment was observed between groupings.

\section{Discussion}

The premise of this study was to test for effect of ILE in toxicity from a $\beta$-blocker of low to moderate lipid solubility. If such an effect were demonstrated, the inference of a metabolic effect for ILE could potentially extend application to alternate agents with similar cardiodepressant properties. The absence of observed effect in this study, when a near identical experiment purported
Table 2 Metoprolol dosing and monitored haemodynamic parameters

\begin{tabular}{|c|c|c|c|}
\hline & Lipid $(n=10)$ & Control $(n=10)$ & $P$ value \\
\hline Metoprolol dosing to toxicity (mg) & $87(46-110)$ & $104(56-145)$ & 0.73 \\
\hline Baseline pulse $\left(\min ^{-1}\right)$ & $223(193-227)$ & $203(185-216)$ & 0.24 \\
\hline Baseline MAP (mmHg) & $79(72-89)$ & $70(58-82)$ & 0.31 \\
\hline Toxicity pulse $\left(\min ^{-1}\right)$ & $193(180-229)$ & $179(163-206)$ & 0.11 \\
\hline Toxicity MAP (mmHg) & $41(40-43)$ & $38(33-40)$ & 0.09 \\
\hline Post-rescue pulse $\left(\min ^{-1}\right)$ & $186(170-216)$ & $180(160-196)$ & 0.31 \\
\hline Post-rescue MAP (mmHg) & $56(50-70)$ & $43(38-54)$ & 0.12 \\
\hline Post-rescue minus tox pulse $\left(\mathrm{min}^{-1}\right)$ & $-9(-10$ to -5$)$ & $-7(-11$ to -5$)$ & 0.71 \\
\hline Post-rescue minus tox MAP (mmHg) & $12(6-26)$ & $7(-1-14)$ & 0.19 \\
\hline 15 min pulse $\left(\min ^{-1}\right)$ & $191(175-220)$ & $182(170-200)$ & 0.38 \\
\hline $15 \min \mathrm{MAP}(\mathrm{mmHg})$ & $59(55-65)$ & $58(52-75)$ & 0.94 \\
\hline
\end{tabular}




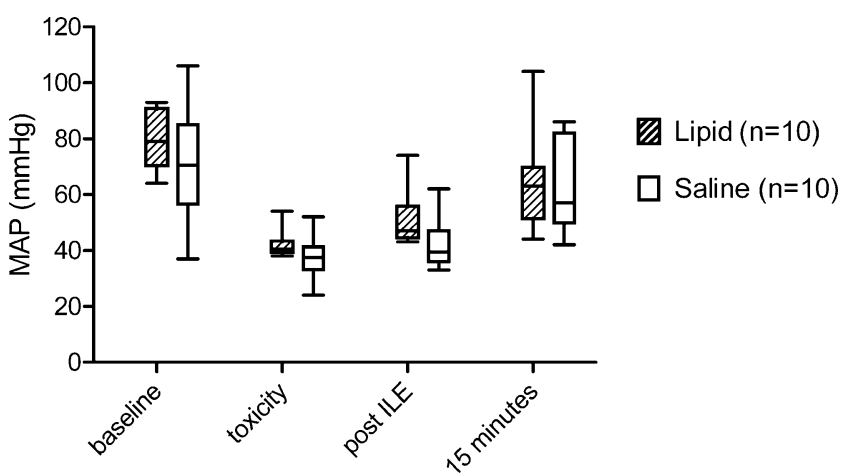

Fig. 1 Mean arterial pressure (MAP) versus time

effect for ILE in toxicity from the highly lipophilic $\beta$ blocker propranolol [10], however, supports to the 'lipid sink' hypothesis over metabolic explanations as the major mechanism for any beneficial effect of ILE in $\beta$-blocker toxicity.

Previous animal investigations support an effect for ILE in propranolol toxicity [8-10]. All three published studies demonstrated significant positive effects for primary or secondary haemodynamic endpoints. Conversely, in the present study, there is no signal from the data to suggest there is any difference in haemodynamic outcome at any time point following ILE administration. It is therefore suggested that drug-lipid sequestration to an expanded intravascular lipid phase and/or augmentation of circulatory carriage of the lipophilic agent to sites of redistribution, not present for metoprolol, may explain the differences observed in ILE efficacy between $\beta$-blockers of disparate lipophilicity.

In the only published animal study to date of toxicity from the hydrophilic $\beta$-blocker atenolol, no effect for ILE was demonstrated on blood pressure or pulse rate in intoxicated rabbits [20]. This study however was thought to have the hypothesis-generating finding of increased rate of improvement in blood pressure early in the course of ILE administration - a finding ascribed by the authors to potential augmentation of fatty acid metabolism. While

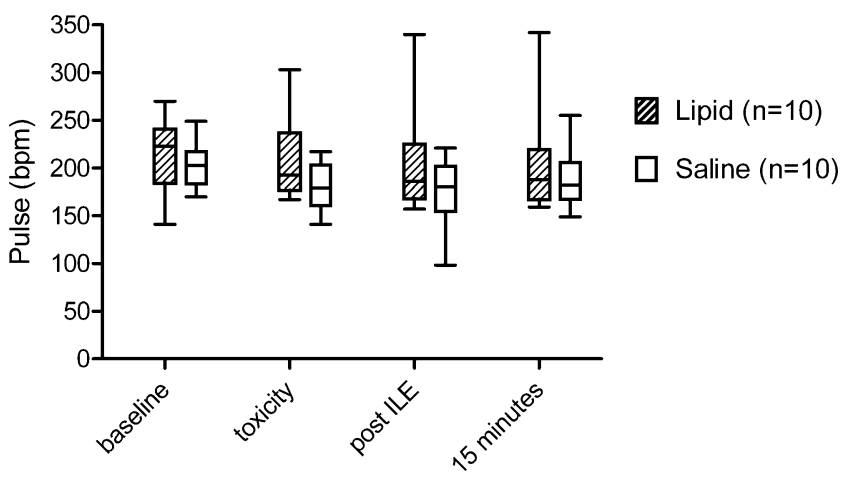

Fig. 2 Heart rate versus time not able to be refuted, this hypothesis is not supported by the results of the present study.

There have been two clinical cases of $\beta$-blocker toxicity treated with ILE reported in the literature. In the first report, a 31-year-old woman presented $4 \mathrm{~h}$ after ingesting approximately $875 \mathrm{mg}(12 \mathrm{mg} / \mathrm{kg})$ carvedilol [21]. She remained shocked despite glucagon, high dose euglycaemic hyperinsulinaemia and maximal dose epinephrine and dopamine infusion. Following $100 \mathrm{ml}$ bolus and $150 \mathrm{ml}$ infusion over $15 \mathrm{~min}$ of $20 \%$ ILE solution, blood pressure was normalised, and vasopressors were weaned allowing extubation. The patient survived.

In the second report, an atenolol toxic patient after initially responding to conventional antidotal treatment (systolic blood pressure (SBP), 96) was treated with ILE over $1 \mathrm{~h}$ and demonstrated further improvement in both SBP and pulse rate [22]. Of the two reports, the carvedilol case seems the more impressive; the patient was still shocked despite maximal conventional therapy yet displayed significant cardiovascular improvement associated with the use of ILE. In the context of available animal data and on review of the presented clinical findings, it would seem likely that ILE was causal in the improvement observed in the former, and less likely responsible for improvement in the latter. Given the lipid solubility of carvedilol is far greater than that of atenolol (Table 3), the outcomes observed in these cases appear to further support the assertion of drug-toxin sequestration as primary mode of action of ILE in the clinical context.

\section{Limitations}

This study is subject to a number of limitations. The inability of this study to demonstrate any difference in haemodynamic parameters between groups may have been in part related to deficiencies in the model of toxicity used. Specifically, our infusion of metoprolol to a defined blood pressure endpoint represents a model for rapidly progressive toxicity and includes no ongoing infusion as mimic for continuous intestinal absorption expected in enteric overdose. In this instance, initial drug redistribution following

Table 3 Lipid solubility (logarithm of solubility coefficients) for various $\beta$-blockers $[30,31]$

\begin{tabular}{lcl}
\hline & $\begin{array}{l}\text { Lipid solubility } \\
\text { coefficient }(\log \mathrm{P})\end{array}$ & $\begin{array}{l}\text { Relative lipid } \\
\text { solubility }\end{array}$ \\
\hline Carvedilol & 3.29 & High \\
Propranolol & 2.80 & High \\
Metoprolol & 1.35 & Low to moderate \\
Atenolol & 0.56 & Low \\
\hline
\end{tabular}


abrupt cessation of infusion may have contributed significantly to the minor observed improvements in MAP, irrespective of treatment employed. The arbitrary endpoint chosen to define toxicity ( $60 \%$ of baseline MAP) may additionally have been insufficiently injurious to demonstrate greater treatment effect. This numeric of toxicity was however elected as previous experiments have demonstrated greater reduction in MAP produced precipitous cardiovascular collapse and animal demise [10]. The decision to monitor post-toxicity for a relatively short period was based on our previous protocols of $\beta$-blocker toxicity. This limits generalisabilty to the clinical scenario, where toxicity often manifests for hours before antidotal therapy. Given that effects were seen at $15 \mathrm{~min}$ in previous protocols [10], this seems unlikely however.

Given the mode of metoprolol extraction, exposure to some other toxic agent cannot be completely excluded as a confounder in the results of the present study. Animals however were uniformly given a pharmacologically available solution of metoprolol and behaved in a manner consistent with $\beta$-blocker toxicity. Additionally, review of the literature [23-26] suggested no excipients in the tablet could have explained these effects.

The appropriate dosing regimen for ILE remains to be elucidated. As such, it is conceivable that an effect for ILE may be demonstrated if greater ILE dosing had been employed and/or if an infusion of ILE had been instituted as in other experimental regimes [1, 2]. Haemodynamic performance in both groups may have also been augmented by the known sympathomimetic effect of ketamine, utilised as sedation. We nonetheless elected to use this agent in line with both institutional protocol and previous work denoting minimal observed effect with ketamine infusion in a similar model of tricyclic antidepressant toxicity [6]. Additionally, the principal investigators in the present study were not blinded to the study intervention; therefore, potential for introduced observer or information bias exists. Primary endpoints were however collated objectively from monitoring equipment in a systematic fashion.

The present study fails to include serum drug levels and detailed pharmacokinetic analysis of metoprolol decay curves, or quantitative assessment of cardiac fatty acid utilisation. As such conclusions around mechanism of action for ILE are based on inference rather than pharmacokinetic data. Furthermore, adverse effects of ILE have not been explored in this study. ILE has been shown to decrease cardiac output and increase pulmonary wedge pressure, when infused into humans undergoing cardiac bypass surgery [27]. Recent case publications following human application, however, show no evidence of this effect $[28,29]$. Finally, the monitoring interval employed in this experiment was relatively short. We are therefore unable to comment on potential late improvement in haemodynamic metrics from either treatment group.
Conclusions from this negative study must be tempered by these adherent methodological limitations. Additionally, and perhaps most importantly, the potential for type 2 error (i.e. falsely negative) is relatively large, equivalent to a $20 \%$ chance of missing an effect comparable to a $25-\mathrm{mmHg}$ increase in MAP in the clinical setting.

\section{Conclusion}

ILE failed to improve immediate haemodynamic parameters in this intact rabbit model of metoprolol toxicity. Care must be taken around negative conclusions drawn from studies of this nature. However, inferential support may be taken for the 'lipid sink' as the explanation for previously observed effects for lipid emulsion in lipophilic $\beta$-blocker toxicity.

Conflict of interest None.

Note A case of intravenous lipid emulsion associated with return of an arrested circulation in nevibolol toxicity has recently been reported [32].

\section{References}

1. Weinberg G, Ripper R, Hoffman W (2003) Lipid emulsion infusion rescues dogs from bupivacaine induced cardiotoxicity. Reg Anesth Pain Med 28:198-202

2. Weinberg G, Di Gregorio G, Ripper R et al (2008) Resuscitation with lipid versus epinephrine in a rat model of bupivacaine overdose. Anesthesiology 108:907-913

3. Tebbutt S, Harvey M, Nicholson T, Cave G (2006) Intralipid prolongs survival in a rat model of verapamil toxicity. Acad Emerg Med 13:134-139

4. Dolcourt B, Aaron C (2008) Intravenous fat emulsion for refractory verapamil and atenolol induced shock: a human case report. Clin Toxicol 46:619-620

5. Yoav G, Odelia G, Shaltiel C (2002) A lipid emulsion reduces mortality from clomipramine overdose in rats. Vet Hum Toxicol 44:30

6. Harvey M, Cave G (2007) Intralipid outperforms sodium bicarbonate in a rabbit model of clomipramine toxicity. Ann Emerg Med 49:178-185

7. Bania T, Chu J (2006) Hemodynamic effect of intralipid in amitriptyline toxicity. Acad Emerg Med 13(S1):117

8. Cave G, Harvey M, Castle C (2006) The role of fat emulsion therapy in a rodent model of propranolol toxicity: a preliminary study. J Med Toxicol 2:4-7

9. Bania T, Chu J, Wesolowski M (2006) The hemodynamic effect of Intralipid on propranolol toxicity. Acad Emerg Med 13(S1):109

10. Harvey M, Cave G (2008) Intralipid infusion ameliorates propranolol induced hypotension in rabbits. J Med Toxicol 4:71-76

11. Matty P, Manu P (2008) Outpatient use of beta blocking agentsprescribing preferences of physicians in training. $\mathrm{J}$ Clin Pharm Therapeut 12:409-414, 6

12. Kerns W (2007) Management of beta adrenergic blocker and calcium channel antagonist toxicity. Emerg Med Clin North Am 25:309-331 
13. O'Grady J, Anderson S, Pringle D (2001) Successful treatment of severe atenolol overdose with calcium chloride. CJEM 3:224-227

14. Weinstein RS (1984) Recognition and management of poisoning with beta-adrenergic blocking agents. Ann Emerg Med 13:1123

15. Crotchety J (1989) Unger: the management of acute poisoning due to beta adrenoreceptor antagonists. Med Toxicol Adverse Drug Exp 4:32-39

16. Kolcz J, Pietrzyk J, Januszewska K, Procelewska M, Mroczek T, Malec E (2007) Extracoporeal life support in severe propranolol and verapamil intoxication. J Intensive Care Med 22:381-385

17. Holger J, Engebretsen K, Fritzlar S, Patten L, Harris C, Flottemesch T (2007) Insulin versus vasopressin and epinephrine to treat beta blocker toxicity. Clin Toxicol 45:396-401

18. Page C, Hacket L, Isbister G (2009) The use of high dose insulin glucose euglycemia in beta blocker overdose: a case report. J Med Toxicol 5:139-143

19. Leppikangas H, Ruokonen E, Rutanen J, Kiviniemi V, Lindgren L, Kurola J (2009) Levosimendan as a rescue drug in experimental propranolol induced myocardial depression: a randomized study. Ann Emerg Med 54:811-817

20. Harvey M, Cave G (2009) Lipid emulsion may augment early blood pressure recovery in a rabbit model of atenolol toxicity. $\mathrm{J}$ Med Toxicol 5:50-51

21. Carr D, Boone A, Hoffman RS, Martin K, Ahluwalia N (2009) Successful resuscitation of a carvedilol overdose using intravenous fat emulsion (IFE). Clin Toxicol 47:727

22. Harchelroad F, Palma A (2008) Efficacy and safety of intravenous lipid therapy in a beta blocker overdose. Clin Toxicol 46:634

23. Lieberman H, Lachman L, Schwartz J (eds) (1990) Pharmaceutical dosage forms: tablets (vol. 1), 2nd edn. Marcel Dekker Inc, New York, p 178
24. Kato H, Kimura K, Izumi S, Nakamichi K, Danjo K, Unada H (2005) The effect of magnesium stearate particle size on tablet properties and tableting characteristics of granules prepared with standard formulation. J Drug Deliv Sci Technol 15:475480

25. Huggins C, Froelich J (1966) High concentration of injected titanium dioxide in abdominal lymph nodes. J Exp Med 124:1099-1110

26. Runge V (2000) Safety of approved MR contrast media for intravenous injection. J Magn Reson Imaging 12:205-213

27. Abel R, Fisch D, Grossman M (1983) Hemodynamic effects of intravenous $20 \%$ soy oil emulsion following coronary bypass surgery. J Parenter Enteral Nutr 7:534-540

28. Rosenblatt M, Abel M, Fischer G, Itkovich C, Eisenkraft J (2006) Successful use of a $20 \%$ lipid emulsion to resuscitate a patient after a presumed bupivacaine-related cardiac arrest. Anesthesiology 105:217-218

29. Litz R, Popp M, Stehr S, Koch T (2006) Successful resuscitation of a patient with ropivacaine-induced asystole after axillary plexus block using lipid infusion. Anaesthesia 61:800-801

30. Petrikova M, Jancinova V, Nosal R, Majekova M (2005) Carvedilol - a $\beta$-blocker with considerable antiaggregatory effect on human blood platelets. Bratisl Lek Listy 106:20-25

31. Lysko P, Webb C, Gu J, Ohlstein E, Ruffolo R, Yue TA (2000) comparison of carvedilol and metoprolol antioxidant properties in vitro. J Cardiovasc Pharmacol 36:277-281

32. Stellpflug SJ, Harris CR, Engebretsen KM, Cole JB, Holger JS (2010) Intentional overdose with cardiac arrest treated with intravenous fat emulsion and high dose insulin. Clin Toxicol, epublished ahead of print 